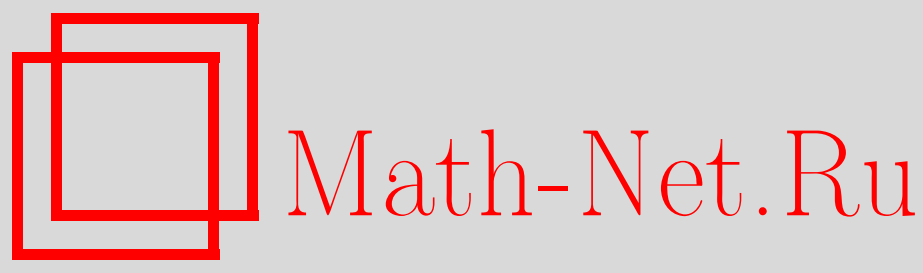

В. В. Жаринов, Симметрии и законы сохранения разностных уравнений, ТМФ, 2011, том 168, номер 2, 195211

DOI: https://doi.org/10.4213/tmf6674

Использование Общероссийского математического портала Math-Net.Ru подразумевает, что вы прочитали и согласны с пользовательским соглашением http://www . mathnet.ru/rus/agreement

Параметры загрузки:

IP : 54.224 .60 .19

26 апреля 2023 г., 09:43:27

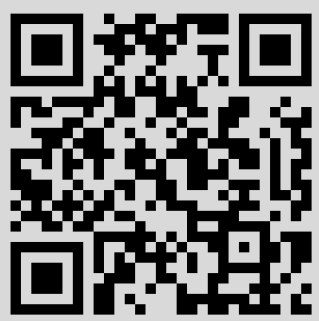




\title{
СИММЕТРИИ И ЗАКОНЫ СОХРАНЕНИЯ РАЗНОСТНЫХ УРАВНЕНИЙ
}

\begin{abstract}
В рамках формализма разностных джетов проводится алгебро-геометрический анализ систем разностных уравнений на многомерных целочисленных решетках, изучаются их симметрии и законы сохранения.
\end{abstract}

Ключевые слова: решетка, разностные уравнения, симметрии, законы сохранения.

\section{1. ВВЕДЕНИЕ}

Алгебро-геометрические методы образуют естественный фундамент для изучения симметрий, законов сохранения, отображений Ли-Беклунда, преобразований Беклунда, интегрируемости и других подобных свойств систем уравнений в частных производных. Эти методы и их приложения подробно изложены в многочисленных монографиях (см., например, [1]-[7]). В последнее время в связи с ростом возможностей компьютерной техники все большую популярность приобретают решеточные модели как для численных расчетов, так и для теоретических исследований в математике и физике. В этом случае также немалый интерес представляют симметрии, законы сохранения и другие геометрические свойства возникающих при этом систем нелинейных разностных уравнений, однако адекватный математический аппарат пока не разработан, хотя имеется немало работ в этом направлении (см., например, [8]-[14]).

В настоящей работе предлагается подход к построению такого аппарата, основанный на понятии разностного джета [15], [16] и позволяющий с единой точки зрения описывать геометрические свойства разностных систем. Отметим, что полученные результаты (теоремы 5-8) существенно отличаются от аналогичных результатов для дифференциальных уравнений. Это связано с принципиальным различием между сдвигами и дифференцированиями: первые задают морфизмы соответствующей алгебры функций, а вторые подчиняются правилу Лейбница на ней.

Будем использовать стандартные обозначения

$$
\mathbb{Z}=\{0, \pm 1, \pm 2, \ldots\}, \quad \mathbb{Z}_{+}=\{0,1,2, \ldots\}, \quad \mathbb{N}=\{1,2,3, \ldots\}
$$

* Математический институт им. В. А. Стеклова РАН, Москва, Россия. E-mail: zharinov@mi.ras.ru 
и обозначать через $\operatorname{Map}(X ; Y)$ множество всех отображений из множества $X$ в множество $Y$. Хотя это явно и не оговаривается, все вводимые ниже пространства обладают естественными топологиями, а все операции предполагаются непрерывными или являются таковыми по построению. В частности, запись $\operatorname{Map}(X ; Y)$ фактически означает пространство всех непрерывных отображений из топологического пространства $X$ в топологическое пространство $Y$, наделенное подходящей топологией.

\section{2. АЛГЕБРО-ГЕОМЕТРИЧЕСКИЙ АНАЛИЗ НА ЦЕЛОЧИСЛЕННОЙ РЕШЕТКЕ}

2.1. Решетка и ее разностный комплекс. Опишем здесь кратко разностный комплекс решетки (подробнее см. [15]-[17]).

Пусть $D \in \mathbb{N}, \mathbf{D}=\{1,2, \ldots, D\}$. Решетка (подробнее, целочисленная решетка) есть множество $\mathbb{L}=\mathbb{Z}^{D}$. На решетке $\mathbb{L}$ определены две согласованные алгебраические структуры - $\mathbb{Z}$-модуля и абелевой группы по сложению. $\mathbb{Z}$-модуль $\mathbb{L}$ имеет базис $\left\{e_{1}, \ldots, e_{D}\right\}$, где

$$
e_{1}=(1,0, \ldots, 0), \quad \ldots, \quad e_{D}=(0, \ldots, 0,1) .
$$

Для $i=\left(i^{1}, \ldots, i^{D}\right)=\sum_{\mu \in \mathbf{D}} i^{\mu} e_{\mu} \in \mathbb{L}$ полагаем $|i|=\sum_{\mu \in \mathbf{D}}\left|i^{\mu}\right| \in \mathbb{Z}_{+}$.

Группа $\mathbb{L}$ действует на решетке $\mathbb{L}$ сдвигами,

$$
T: \mathbb{L} \rightarrow \operatorname{Aut}(\mathbb{L}), \quad i \mapsto T_{i}: \mathbb{L} \rightarrow \mathbb{L}, \quad k \mapsto T_{i} k=k+i .
$$

На алгебре $\mathcal{A}(\mathbb{L})=\operatorname{Map}(\mathbb{L} ; \mathbb{R})$ группа $\mathbb{L}$ действует по правилу

$$
T: \mathbb{L} \rightarrow \operatorname{Aut}(\mathcal{A}(\mathbb{L})), \quad i \mapsto T_{i}: \mathcal{A}(\mathbb{L}) \rightarrow \mathcal{A}(\mathbb{L}), \quad \varphi \mapsto T_{i} \varphi=\varphi \circ T_{i},
$$

так что $\left(T_{i} \varphi\right)(k)=\varphi\left(T_{i} k\right)=\varphi(k+i)$ для всех $\varphi \in \mathcal{A}(\mathbb{L}), i, k \in \mathbb{L}$.

Определен $\mathcal{A}(\mathbb{L})$-модуль

$$
\mathcal{D}(\mathbb{L})=\left\{\zeta=\sum_{\mu \in \mathbf{D}} \zeta^{\mu} \cdot \Delta_{\mu} \mid \zeta^{\mu} \in \mathcal{A}(\mathbb{L})\right\} \subset \operatorname{End}_{\mathbb{R}}(\mathcal{A}(\mathbb{L}))
$$

с базисом $\left\{\Delta_{1}, \ldots, \Delta_{D}\right\}$, где элементарные разностные операторы

$$
\Delta_{\mu}=T_{e_{\mu}}-T_{0} \in \operatorname{End}_{\mathbb{R}}(\mathcal{A}(\mathbb{L})), \quad \mu \in \mathbf{D} .
$$

Дуальный $\mathcal{A}(\mathbb{L})$-модуль

$$
\mathcal{D}^{*}(\mathbb{L})=\operatorname{Hom}_{\mathcal{A}(\mathbb{L})}(\mathcal{D}(\mathbb{L}) ; \mathcal{A}(\mathbb{L}))=\left\{\varrho=\sum_{\mu \in \mathbf{D}} \varrho_{\mu} \cdot \Delta^{\mu} \mid \varrho_{\mu} \in \mathcal{A}(\mathbb{L})\right\}
$$

имеет дуальный базис $\left\{\Delta^{1}, \ldots, \Delta^{D}\right\}$, где $\left\langle\Delta^{\mu}, \Delta_{\nu}\right\rangle=\delta_{\nu}^{\mu}$ для всех $\mu, \nu \in \mathbf{D}$. Группа $\mathbb{L}$ действует на линейных пространствах $\mathcal{D}(\mathbb{L})$ и $\mathcal{D}^{*}(\mathbb{L})$ покомпонентно,

$$
\begin{aligned}
T: \mathbb{L} \rightarrow \operatorname{Aut}(\mathcal{D}(\mathbb{L})), & i \mapsto T_{i}: \mathcal{D}(\mathbb{L}) \rightarrow \mathcal{D}(\mathbb{L}), \\
\zeta & =\sum_{\mu} \zeta^{\mu} \cdot \Delta_{\mu} \mapsto T_{i} \zeta=\sum_{\mu}\left(T_{i} \zeta^{\mu}\right) \cdot \Delta_{\mu}, \\
T: \mathbb{L} \rightarrow \operatorname{Aut}\left(\mathcal{D}^{*}(\mathbb{L})\right), & i \mapsto T_{i}: \mathcal{D}^{*}(\mathbb{L}) \rightarrow \mathcal{D}^{*}(\mathbb{L}), \\
\varrho & =\sum_{\mu} \varrho_{\mu} \cdot \Delta^{\mu} \mapsto T_{i} \varrho=\sum_{\mu}\left(T_{i} \varrho_{\mu}\right) \cdot \Delta^{\mu},
\end{aligned}
$$


так что

$$
T_{i}\langle\varrho, \zeta\rangle=T_{i}\left(\sum_{\mu} \varrho_{\mu} \zeta^{\mu}\right)=\sum_{\mu}\left(T_{i} \varrho_{\mu}\right)\left(T_{i} \zeta^{\mu}\right)=\left\langle T_{i} \varrho, T_{i} \zeta\right\rangle
$$

для всех $i \in \mathbb{L}, \varrho \in \mathcal{D}^{*}(\mathbb{L}), \zeta \in \mathcal{D}(\mathbb{L})$.

Определена градуированная внешняя алгебра

$$
\Omega(\mathbb{L})=\bigoplus_{0 \leqslant q \leqslant D} \Omega^{q}(\mathbb{L})
$$

где $\mathcal{A}(\mathbb{L})$-модули

$$
\begin{aligned}
\Omega^{q}(\mathbb{L}) & =\operatorname{Hom}_{\mathcal{A}(\mathbb{L})}\left(\bigwedge_{\mathcal{A}(\mathbb{L})}^{q} \mathcal{D}(\mathbb{L}) ; \mathcal{A}(\mathbb{L})\right)=\bigwedge_{\mathcal{A}(\mathbb{L})}^{q} \mathcal{D}^{*}(\mathbb{L})= \\
& =\left\{\varrho=\frac{1}{q !} \sum_{m \in \mathbf{D}_{q}} \varrho_{m} \cdot \Delta^{m} \mid \varrho_{m} \in \mathcal{A}(\mathbb{L})\right\},
\end{aligned}
$$

$m=\left(\mu_{1}, \ldots, \mu_{q}\right), \Delta^{m}=\Delta^{\mu_{1}} \wedge \cdots \wedge \Delta^{\mu_{q}}$. В частности, $\Omega^{0}(\mathbb{L})=\mathcal{A}(\mathbb{L}), \Omega^{1}(\mathbb{L})=\mathcal{D}^{*}(\mathbb{L})$.

Действие группы $\mathbb{L}$ на градуированной внешней алгебре $\Omega(\mathbb{L})$ определяется ее действием на линейных пространствах $\mathcal{A}(\mathbb{L}), \mathcal{D}^{*}(\mathbb{L})$ и условием $T_{i}(\varrho \wedge \chi)=\left(T_{i} \varrho\right) \wedge$ $\left(T_{i} \chi\right)$ для всех $i \in \mathbb{L}, \varrho, \chi \in \Omega(\mathbb{L})$, т. е. по-прежнему покомпонентное.

Определен разностный дифференциал

$$
d \in \operatorname{End}_{\mathbb{R}}(\Omega(\mathbb{L})), \quad \varrho \mapsto d \varrho=\sum_{\mu \in \mathbf{D}} \Delta^{\mu} \wedge \Delta_{\mu} \varrho,
$$

где элементарные разностные операторы

$$
\Delta_{\mu}=T_{e_{\mu}}-T_{0} \in \operatorname{End}_{\mathbb{R}}(\Omega(\mathbb{L})), \quad \mu \in \mathbf{D},
$$

причем по построению

a) $d \circ d=0$

б) $d^{q}=\left.d\right|_{\Omega^{q}(\mathbb{L})}: \Omega^{q}(\mathbb{L}) \rightarrow \Omega^{q+1}(\mathbb{L})$ для всех $0 \leqslant q \leqslant D$.

Таким образом, определен комплекс линейных пространств $\left\{\Omega^{q}(\mathbb{L}) ; d^{q}\right\}-$ pазностный комплекс решетки $\mathbb{L}$.

Теорема 1. Пополненный разностный комплекс решетки $\mathbb{L}$

$$
0 \longrightarrow \mathbb{R} \longrightarrow \Omega^{0}(\mathbb{L}) \stackrel{d}{\longrightarrow} \Omega^{1}(\mathbb{L}) \stackrel{d}{\longrightarrow} \cdots \stackrel{d}{\longrightarrow} \Omega^{D}(\mathbb{L}) \longrightarrow 0
$$

точный, т.е. все его пространства когомологий тривиальные.

2.2. Расслоение разностных джетов. Изложим кратко основы аппарата разностных джетов (подробнее см. [15], [16]).

Пусть $V$ - линейное пространство, на котором группа $\mathbb{L}$ действует тривиально,

$$
T: \mathbb{L} \rightarrow \operatorname{Aut}(V), \quad i \mapsto T_{i}: V \rightarrow V, \quad u \mapsto T_{i} u=u .
$$

Будем рассматривать прямое произведение $J=\mathbb{L} \times V$ как векторное расслоение над решеткой $\mathbb{L}$ с типичным слоем $V$ и проекцией на первый сомножитель

$$
\pi: J=\mathbb{L} \times V \rightarrow \mathbb{L}, \quad(k, u) \mapsto \pi(k, u)=k .
$$


Поскольку, группа $\mathbb{L}$ действует на слоях тривиально, ее действие на расслоении $\pi$ имеет вид

$$
T: \mathbb{L} \rightarrow \operatorname{Aut}(\pi), \quad i \mapsto T_{i}: J \rightarrow J, \quad(k, u) \mapsto T_{i}(k, u)=\left(T_{i} k, u\right),
$$

подробнее $T_{i}=\left(T_{i}, T_{i}\right) \in \operatorname{Aut}(\mathbf{J}) \times \operatorname{Aut}(\mathbb{L})$, где $T_{i}$ о $\pi=\pi \circ T_{i}$, причем $T_{i}(k, u)=(k+i, u)$, $T_{i} k=k+i$ для всех $i, k \in \mathbb{L}, u \in V$. Индуцированное действие на $\mathcal{A}(\mathbb{L})$-модуле

$$
\mathcal{M}(\pi)=\left\{\varphi \in \operatorname{Map}(\mathbb{L} ; J) \mid \pi \circ \varphi=\operatorname{id}_{\mathbb{L}}\right\}=\operatorname{Map}(\mathbb{L} ; V)
$$

всех сечений расслоения $\pi: J \rightarrow \mathbb{L}$ имеет вид

$$
T: \mathbb{L} \rightarrow \operatorname{Aut}(\mathcal{M}(\pi)), \quad i \mapsto T_{i}: \mathcal{M}(\pi) \rightarrow \mathcal{M}(\pi), \quad \varphi \mapsto T_{i} \varphi=\varphi \circ T_{i}
$$

так что $\left(T_{i} \varphi\right)(k)=\varphi(k+i)$ для всех $i, k \in \mathbb{L}, \varphi \in \mathcal{M}(\pi)$.

Расслоение $\pi$ индуцирует расслоение разностных джетов

$$
\boldsymbol{\pi}: \mathbf{J}=\mathbb{L} \times \mathbf{V} \rightarrow \mathbb{L}, \quad(k, \mathbf{u}) \mapsto \boldsymbol{\pi}(k, \mathbf{u})=k,
$$

над решеткой $\mathbb{L}$, где типичный слой

$$
\mathbf{V}=\left\{\mathbf{u}=\left(u_{l}\right) \mid l \in \mathbb{L}, u_{l} \in V\right\}=V_{\mathbb{L}} \simeq \mathcal{M}(\pi) .
$$

Действие группы $\mathbb{L}$ на типичном слое $\mathbf{V}$ определяется согласно ее действию на $\mathcal{M}(\pi)$,

$$
T: \mathbb{L} \rightarrow \operatorname{Aut}(\mathbf{V}), \quad i \mapsto T_{i}: \mathbf{V} \rightarrow \mathbf{V}, \quad \mathbf{u} \mapsto T_{i} \mathbf{u}, \quad\left(T_{i} \mathbf{u}\right)_{l}=u_{l+i}, \quad l \in \mathbb{L} .
$$

Соответственно, действие группы $\mathbb{L}$ на расслоении $\boldsymbol{\pi}$ имеет вид

$$
T: \mathbb{L} \rightarrow \operatorname{Aut}(\boldsymbol{\pi}), \quad i \mapsto T_{i}: \mathbf{J} \rightarrow \mathbf{J}, \quad(k, \mathbf{u}) \mapsto T_{i}(k, \mathbf{u})=\left(T_{i} k, T_{i} \mathbf{u}\right),
$$

подробнее $T_{i}=\left(T_{i}, T_{i}\right) \in \operatorname{Aut}(\mathbf{J}) \times \operatorname{Aut}(\mathbb{L})$, где $T_{i} \circ \boldsymbol{\pi}=\boldsymbol{\pi} \circ T_{i}$, причем $T_{i}(k, \mathbf{u})=$ $\left(k+i, T_{i} \mathbf{u}\right), T_{i} k=k+i$ для всех $i, k \in \mathbb{L}, \mathbf{u} \in \mathbf{V}$. Индуцированное действие на $\mathcal{A}(\mathbb{L})$-модуле

$$
\mathcal{M}(\boldsymbol{\pi})=\left\{\boldsymbol{\phi} \in \operatorname{Map}(\mathbb{L} ; \mathbf{J}) \mid \boldsymbol{\pi} \circ \boldsymbol{\phi}=\operatorname{id}_{\mathbb{L}}\right\}=\operatorname{Map}(\mathbb{L} ; \mathbf{V})
$$

всех сечений расслоения $\boldsymbol{\pi}: \mathbf{J} \rightarrow \mathbb{L}$ имеет вид

$$
T: \mathbb{L} \rightarrow \operatorname{Aut}(\mathcal{M}(\boldsymbol{\pi})), \quad i \mapsto T_{i}: \mathcal{M}(\boldsymbol{\pi}) \rightarrow \mathcal{M}(\boldsymbol{\pi}), \quad \phi \mapsto T_{i} \phi,
$$

где $T_{i} \boldsymbol{\phi}=T_{-i} \circ \boldsymbol{\phi} \circ T_{i}$, так что $\left(T_{i} \phi\right)_{l}(k)=\varphi_{l-i}(k+i)$ для всех $\boldsymbol{\phi}=\left(\varphi_{l}\right) \in \mathcal{M}(\boldsymbol{\pi})$, $i, l, k \in \mathbb{L}$.

Определено линейное подпространство

$$
\begin{aligned}
\mathcal{M}_{T}(\boldsymbol{\pi}) & =\left\{\boldsymbol{\phi} \in \mathcal{M}(\boldsymbol{\pi}) \mid T_{i} \boldsymbol{\phi}=\boldsymbol{\phi} \text { для всех } i \in \mathbb{L}\right\}= \\
& =\left\{\boldsymbol{\phi} \in \mathcal{M}(\boldsymbol{\pi}) \mid T_{i} \circ \boldsymbol{\phi}=\boldsymbol{\phi} \circ T_{i} \text { для всех } i \in \mathbb{L}\right\}
\end{aligned}
$$

всех инвариантных сечений (подробнее сечений, инвариантных относительно сдвигов) расслоения $\boldsymbol{\pi}: \mathbf{J} \rightarrow \mathbb{L}$.

Определено отображение

$$
\mathbf{j}: \mathcal{M}(\pi) \rightarrow \mathcal{M}(\boldsymbol{\pi}), \quad \varphi \mapsto \mathbf{j} \varphi, \quad(\mathbf{j} \varphi)_{l}=T_{l} \varphi, \quad l \in \mathbb{L},
$$

подробнее $(\mathbf{j} \varphi)_{l}(k)=\varphi(k+l)$ для всех $\varphi \in \mathcal{M}(\pi), l, k \in \mathbb{L}$. 
ПреДлОЖЕнИЕ 1. Справедливы следующие утверждения.

1. Отображение $\mathbf{j}: \mathcal{M}(\pi) \rightarrow \mathcal{M}(\boldsymbol{\pi})$ ингективное. Более того, если джеты $\mathbf{j} \varphi, \mathbf{j} \psi \in \mathcal{M}(\boldsymbol{\pi})$ совпадают хотя бъ в одной точке $k \in \mathbb{L}$, то сечения $\varphi, \psi \in \mathcal{M}(\pi)$ совпадают на всей решетке $\mathbb{L}$.

2. Имеет место изоморфизм

$$
\mathbf{j}: \mathcal{M}(\pi) \simeq \mathcal{M}_{T}(\boldsymbol{\pi}) .
$$

2.3. Инвариантные подмножества и сечения. Пусть $\pi: \mathbf{J}=\mathbb{L} \times \mathbf{V} \rightarrow \mathbb{L}-$ расслоение разностных джетов над решеткой $\mathbb{L}$. По построению:

a) для каждого $k \in \mathbb{L}$ имеет место изоморфизм

$$
\boldsymbol{\pi}^{-1}(k) \simeq \mathbf{V}, \quad(k, \mathbf{u}) \mapsto \mathbf{u},
$$

б) $T_{i} \boldsymbol{\pi}^{-1}(k)=\boldsymbol{\pi}^{-1}\left(T_{i} k\right)=\boldsymbol{\pi}^{-1}(k+i)$ для всех $i, k \in \mathbb{L}$.

Для подмножества $\mathbf{F} \subset \mathbf{J}$ и точки $k \in \mathbb{L}$ положим

$$
\mathbf{F}_{k}=\mathbf{F} \cap \boldsymbol{\pi}^{-1}(k) \quad \text { и } \quad \mathcal{F}_{k}=\left\{\mathbf{u} \in \mathbf{V} \mid(k, \mathbf{u}) \in \mathbf{F}_{k}\right\},
$$

так что $\mathbf{F}_{k} \simeq \mathcal{F}_{k},(k, \mathbf{u}) \mapsto \mathbf{u}$.

Подмножество $\mathbf{F} \subset \mathbf{J}$ будем называть инвариантным (подробнее, инвариантным относительно сдвигов), если $T_{i} \mathbf{F}=\mathbf{F}$ для всех $i \in \mathbb{L}$.

Тривиальный пример инвариантного подмножества есть $\mathbf{F}=\mathbf{J}$.

ПРЕДЛОЖеНИЕ 2. Пусть подмножество $\mathbf{F} \subset \mathbf{J}$. Следующие утверждения эквивалентны:

1) подмножество $\mathbf{F}$ инвариантное;

2) действие $T: \mathbb{L} \rightarrow \operatorname{Aut}(\mathbf{J})$ имеет сужение

$$
T=\left.T\right|_{\mathbf{F}}: \mathbb{L} \rightarrow \operatorname{Aut}(\mathbf{F}), \quad i \mapsto T_{i}=\left.T_{i}\right|_{\mathbf{F}}: \mathbf{F} \rightarrow \mathbf{F}, \quad(k, \mathbf{u}) \mapsto\left(T_{i} k, T_{i} \mathbf{u}\right) ;
$$

3) для всех $i, k \in \mathbb{L}$ имеют место изоморфизмы $T_{i}: \mathcal{F}_{k} \simeq \mathcal{F}_{k+i}$;

4) имеет место изоморфизм

$$
\varkappa: \mathbf{F} \simeq \widetilde{\mathbf{F}}=\mathbb{L} \times \mathcal{F}, \quad(k, \mathbf{u}) \mapsto \varkappa(k, \mathbf{u})=(k, \tilde{\mathbf{u}}), \quad \tilde{\mathbf{u}}=T_{-k} \mathbf{u},
$$

здесь и всюоу ниже $\mathcal{F}=\mathcal{F}_{0} \subset \mathbf{V}$.

ДоКАЗАТЕЛЬСТВО проводится прямой проверкой.

В частности, для каждого инвариантного подмножества $\mathbf{F}$ пространства $\mathbf{J}$ определено подрасслоение $\boldsymbol{\pi}_{\mathbf{F}}=\left.\boldsymbol{\pi}\right|_{\mathbf{F}}: \mathbf{F} \rightarrow \mathbb{L}$ расслоения $\boldsymbol{\pi}: \mathbf{J} \rightarrow \mathbb{L}$ с типичным слоем $\mathcal{F}$, на котором определено действие

$$
T: \mathbb{L} \rightarrow \operatorname{Aut}\left(\boldsymbol{\pi}_{\mathbf{F}}\right), \quad i \mapsto T_{i}: \mathbf{F} \rightarrow \mathbf{F}, \quad(k, \mathbf{u}) \mapsto T_{i}(k, \mathbf{u})=\left(T_{i} k, T_{i} \mathbf{u}\right) .
$$

Для каждого инвариантного подмножества $\mathbf{F} \subset \mathbf{J}$ определены следующие множества:

a) $\mathcal{M}\left(\boldsymbol{\pi}_{\mathbf{F}}\right)=\left\{\phi \in \operatorname{Map}(\mathbb{L} ; \mathbf{F}) \mid \boldsymbol{\pi}_{\mathbf{F}} \circ \phi=\operatorname{id}_{\mathbb{L}}\right\}=\{\phi \in \mathcal{M}(\boldsymbol{\pi}) \mid \operatorname{graph} \phi \subset \mathbf{F}\}-$ множество всех сечений подрасслоения $\boldsymbol{\pi}_{\mathbf{F}}: \mathbf{F} \rightarrow \mathbb{L}$, где graph $\boldsymbol{\phi}$ - график сечения $\boldsymbol{\phi} ;$

б) $\mathcal{M}_{T}\left(\boldsymbol{\pi}_{\mathbf{F}}\right)=\mathcal{M}_{T}(\boldsymbol{\pi}) \cap \mathcal{M}\left(\boldsymbol{\pi}_{\mathbf{F}}\right)$ - множество всех инвариантных сечений подрасслоения $\boldsymbol{\pi}_{\mathbf{F}}: \mathbf{F} \rightarrow \mathbb{L}$;

в) $\mathcal{M}_{\mathbf{F}}(\pi)=\left\{\varphi \in \mathcal{M}(\pi) \mid \mathbf{j} \varphi \in \mathcal{M}\left(\boldsymbol{\pi}_{\mathbf{F}}\right)\right\}$ - множество всех сечений расслоения $\pi: J \rightarrow \mathbb{L}$, допустимых для подрасслоения $\boldsymbol{\pi}_{\mathrm{F}}: \mathbf{F} \rightarrow \mathbb{L}$. 
ПрЕДЛОЖЕНИЕ 3. Пусть $\mathbf{F}$ - инвариантное подмножество пространства $\mathbf{J}$. Тогда справедливы следующие утверждения.

1. Для каждой точки $(k, \mathbf{u}) \in \mathbf{F}$ существует одно и только одно допустимое сечение $\varphi \in \mathcal{M}_{\mathbf{F}}(\pi)$ такое, ито $(\mathbf{j}(\varphi))(k)=\mathbf{u}$.

2. $\mathcal{M}_{\mathbf{F}}(\pi) \simeq \mathcal{M}_{T}\left(\boldsymbol{\pi}_{\mathbf{F}}\right) \simeq \mathcal{F}=\mathcal{F}_{0}, \varphi \mapsto \mathbf{j} \varphi, \phi \mapsto \phi(0)$, в первом случае $\varphi=(\varphi(k)) \mapsto$ $\boldsymbol{\phi}=\left(\varphi_{l}(k)\right), \varphi_{l}(k)=\varphi(k+l)$, а во втором случае $\boldsymbol{\phi}=\left(\varphi_{l}(k)\right) \mapsto \mathbf{u}=\left(u_{l}\right), u_{l}=\varphi_{l}(0)$. В частности, $\varphi=(\varphi(k)) \mapsto \mathbf{u}=\left(u_{l}\right), u_{l}=\varphi(l)$.

ДокАЗАТЕЛЬСтво. Для данной точки $(k, \mathbf{u}) \in \mathbf{F}$ сечение $\varphi \in \mathcal{M}(\mathbb{L}, V)$ зададим правилом $\varphi(i)=u_{i-k}$ для всех $i \in \mathbb{L}$. Тогда по построению $(\mathbf{j} \varphi)_{l}(i)=u_{i+l-k}$ для всех $l, i \in \mathbb{L}$. В частности, $(\mathbf{j} \varphi)_{l}(k)=u_{l}$ для всех $l \in \mathbb{L}$, т. е. $(\mathbf{j} \varphi)(k)=\mathbf{u} \in \mathcal{F}_{k}$. Кроме того, $(\mathbf{j} \varphi)_{l}(i)=u_{i+l-k}=\left(T_{i-k} \mathbf{u}\right)_{l}$ для всех $l, i \in \mathbb{L}$, так что $(\mathbf{j} \varphi)(i)=T_{i-k} \mathbf{u} \in T_{i-k} \mathcal{F}_{k}=$ $\mathcal{F}_{i}$ в силу инвариантности подмножества $\mathbf{F}$ (см. предложение 2). Таким образом, построенное сечение $\varphi$ допустимое для $\mathbf{F}$. Единственность такого сечения следует из предложения 1 , так что первое утверждение доказано. Второе утверждение следует из первого и предложения 1.

2.4. Симметрии. Пусть $\mathbf{F}$ - инвариантное подмножество пространства $\mathbf{J}$. Группа

$$
\operatorname{Aut}\left(\boldsymbol{\pi}_{\mathbf{F}}\right)=\left\{R=\left(R_{\mathbf{F}}, R_{\mathbb{L}}\right) \in \operatorname{Aut}(\mathbf{F}) \times \operatorname{Aut}(\mathbb{L}) \mid \boldsymbol{\pi}_{\mathbf{F}} \circ R_{\mathbf{F}}=R_{\mathbb{L}} \circ \boldsymbol{\pi}_{\mathbf{F}}\right\}
$$

всех автоморфизмов расслоения $\boldsymbol{\pi}_{\mathbf{F}}: \mathbf{F} \rightarrow \mathbb{L}$ имеет подгруппу

$$
\operatorname{Aut}_{T}\left(\boldsymbol{\pi}_{\mathbf{F}}\right)=\left\{R \in \operatorname{Aut}\left(\boldsymbol{\pi}_{\mathbf{F}}\right) \mid T_{i} \circ R=R \circ T_{i} \text { для всех } i \in \mathbb{L}\right\}
$$

всех инвариантных автоморфизмов расслоения $\boldsymbol{\pi}_{\mathbf{F}}: \mathbf{F} \rightarrow \mathbb{L}$ (поясним, что здесь $\left.T_{i}=\left(T_{i \mathbf{F}}, T_{i \mathbb{L}}\right) \in \operatorname{Aut}\left(\boldsymbol{\pi}_{\mathbf{F}}\right)\right)$. B свою очередь, группа $\mathbb{L}$ порождает подгруппу $\mathcal{T}=$ $\left\{T_{i} \in \operatorname{Aut}\left(\boldsymbol{\pi}_{\mathbf{F}}\right) \mid i \in \mathbb{L}\right\}$ группы $\operatorname{Aut}_{T}\left(\boldsymbol{\pi}_{\mathbf{F}}\right)$. Эта подгруппа нормальна, поскольку по построению $T_{i} \circ R=R \circ T_{i}$ для всех $i \in \mathbb{L}$ и $R \in \operatorname{Aut}_{T}\left(\boldsymbol{\pi}_{\mathbf{F}}\right)$, так что определена факторгруппа

$$
\operatorname{SYM}\left(\boldsymbol{\pi}_{\mathbf{F}}\right)=\operatorname{Aut}_{T}\left(\boldsymbol{\pi}_{\mathbf{F}}\right) / \mathcal{T}=\left\{\mathbf{R}=R \circ \mathcal{T} \mid R \in \operatorname{Aut}_{T}\left(\boldsymbol{\pi}_{\mathbf{F}}\right)\right\}
$$

симметрий расслоения $\boldsymbol{\pi}_{\mathbf{F}}: \mathbf{F} \rightarrow \mathbb{L}$.

ПрЕДЛОЖЕНИЕ 4. Пусть $\mathbf{F}$ - инвариантное подмножество пространства $\mathbf{J}$. Группа автоморфизмов $\operatorname{Aut}\left(\boldsymbol{\pi}_{\mathbf{F}}\right)$ действует на множестве сечений $\mathcal{M}\left(\boldsymbol{\pi}_{\mathbf{F}}\right)$ по правилу

$$
\begin{aligned}
\operatorname{Aut}\left(\boldsymbol{\pi}_{\mathbf{F}}\right) \rightarrow \operatorname{Aut}\left(\mathcal{M}\left(\boldsymbol{\pi}_{\mathbf{F}}\right)\right), \quad R \mapsto R: \mathcal{M}\left(\boldsymbol{\pi}_{\mathbf{F}}\right) & \rightarrow \mathcal{M}\left(\boldsymbol{\pi}_{\mathbf{F}}\right), \\
\boldsymbol{\phi} & \mapsto R \phi=R^{-1} \circ \phi \circ R
\end{aligned}
$$

(подробнее, $\left.R \boldsymbol{\phi}=R_{\mathbf{F}}^{-1} \circ \phi \circ R_{\mathbb{L}}\right)$. Это действие имеет сужение

$$
\operatorname{Aut}_{T}\left(\boldsymbol{\pi}_{\mathbf{F}}\right) \rightarrow \operatorname{Aut}\left(\mathcal{M}_{T}\left(\boldsymbol{\pi}_{\mathbf{F}}\right)\right)
$$

ДокАЗАтЕЛЬСтво. Действительно, пусть $R \in \operatorname{Aut}\left(\boldsymbol{\pi}_{\mathbf{F}}\right), \boldsymbol{\pi}_{\mathbf{F}} \circ R=R \circ \boldsymbol{\pi}_{\mathbf{F}}$, и $\boldsymbol{\phi} \in$ $\mathcal{M}\left(\boldsymbol{\pi}_{\mathbf{F}}\right), \boldsymbol{\pi}_{\mathbf{F}} \circ \boldsymbol{\phi}=\mathrm{id}_{\mathbb{L}}$. Тогда

$\boldsymbol{\pi}_{\mathbf{F}} \circ R \phi=\pi_{\mathbf{F}} \circ R^{-1} \circ \phi \circ R=R^{-1} \circ \boldsymbol{\pi}_{\mathbf{F}} \circ \phi \circ R=R^{-1} \circ \mathrm{id}_{\mathbb{L}} \circ R=R^{-1} \circ R=\mathrm{id}_{\mathbb{L}}$. 
Если, кроме того, $T_{i} \circ R=R \circ T_{i}$ и $T_{i} \circ \phi=\phi \circ T_{i}, i \in \mathbb{L}$, то

$$
T_{i} \circ R \phi=T_{i} \circ R^{-1} \circ \phi \circ R=R^{-1} \circ T_{i} \circ \phi \circ T_{i} \circ R=R^{-1} \circ \phi \circ R \circ T_{i}=R \phi \circ T_{i} .
$$

СлеДСТвИЕ 1. Пусть $\mathbf{F}$ - инвариантное подмножество пространства Ј. Дейcтвие $\operatorname{Aut}_{T}\left(\boldsymbol{\pi}_{\mathbf{F}}\right) \rightarrow \operatorname{Aut}\left(\mathcal{M}_{T}\left(\boldsymbol{\pi}_{\mathbf{F}}\right)\right)$ индуцирует соответствующее действие на множестве допустимых сечений $\mathcal{M}_{\mathbf{F}}(\pi)$,

$$
\operatorname{Aut}_{T}\left(\boldsymbol{\pi}_{\mathbf{F}}\right) \rightarrow \operatorname{Aut}\left(\mathcal{M}_{\mathbf{F}}(\pi)\right), \quad R \mapsto R_{*}=\mathbf{j}^{-1} \circ R \circ \mathbf{j} .
$$

ДокАЗАТЕЛЬСтво. Достаточно воспользоваться предложениями 3 и 4.

По определению $T_{i} \phi=\phi$ для всех $i \in \mathbb{L}$ и $\phi \in \mathcal{M}_{T}\left(\boldsymbol{\pi}_{\mathbf{F}}\right)$, т. е. нормальная подгруппа $\mathcal{T}$ действует на множестве $\mathcal{M}_{T}\left(\boldsymbol{\pi}_{\mathbf{F}}\right)$ тривиальным образом, так что определено фактор-действие

$$
\begin{aligned}
\operatorname{SYM}\left(\boldsymbol{\pi}_{\mathbf{F}}\right) \rightarrow \operatorname{Aut}\left(\mathcal{M}_{T}\left(\boldsymbol{\pi}_{\mathbf{F}}\right)\right), \quad \mathbf{R} \mapsto \mathbf{R}: \mathcal{M}_{T}\left(\boldsymbol{\pi}_{\mathbf{F}}\right) & \rightarrow \mathcal{M}_{T}\left(\boldsymbol{\pi}_{\mathbf{F}}\right), \\
\boldsymbol{\phi} & \mapsto \mathbf{R} \boldsymbol{\phi}=R \boldsymbol{\phi} .
\end{aligned}
$$

Как и выше, это действие индуцирует действие на множестве $\mathcal{M}_{\mathbf{F}}(\pi)$,

$$
\operatorname{SYM}\left(\boldsymbol{\pi}_{\mathbf{F}}\right) \rightarrow \operatorname{Aut}\left(\mathcal{M}_{\mathbf{F}}(\pi)\right), \quad \mathbf{R} \mapsto \mathbf{R}_{*}=\mathbf{j}^{-1} \circ \mathbf{R} \circ \mathbf{j},
$$

что позволяет считать факторгруппу $\operatorname{SYM}\left(\boldsymbol{\pi}_{\mathbf{F}}\right)$ также и группой симметрий множества $\mathcal{M}_{\mathbf{F}}(\pi)$ допустимых сечений расслоения $\boldsymbol{\pi}_{\mathbf{F}}: \mathbf{F} \rightarrow \mathbb{L}$.

Изучим группу $\operatorname{SYM}\left(\boldsymbol{\pi}_{\mathbf{F}}\right)$ подробнее.

Сначала отметим, что группы $\operatorname{Aut}\left(\mathcal{F}_{k}\right)$ автоморфизмов множеств $\mathcal{F}_{k}, k \in \mathbb{L}$, образуют главное расслоение над $\mathbb{L}$ со структурной группой $\operatorname{Aut}(\mathcal{F})$ (напомним, что $\left.\mathcal{F}=\mathcal{F}_{0}\right)$

$$
\mathbf{\Pi}: \mathbf{A F}=\bigcup_{k \in \mathbb{L}} \operatorname{Aut}\left(\mathcal{F}_{k}\right) \rightarrow \mathbb{L}, \quad \mathbf{r}_{k} \mapsto \mathbf{\Pi}\left(\mathbf{r}_{k}\right)=k,
$$

поскольку равенства $T_{i} \mathcal{F}_{k}=\mathcal{F}_{k+i}, i, k \in \mathbb{L}$ (см. предложение 2$)$, порождают изоморфизмы

$$
T_{i}: \operatorname{Aut}\left(\mathcal{F}_{k}\right) \simeq \operatorname{Aut}\left(\mathcal{F}_{k+i}\right), \quad \mathbf{r}_{k} \mapsto T_{i} \mathbf{r}_{k}=T_{i} \circ \mathbf{r}_{k} \circ T_{-i},
$$

так что определен изоморфизм расслоений над $\mathbb{L}$

$$
\mathbf{A F} \simeq \mathbb{L} \times \operatorname{Aut}(\mathcal{F}), \quad \mathbf{r}_{k} \mapsto(k, \boldsymbol{\rho}), \quad \boldsymbol{\rho}=T_{-k} \circ \mathbf{r}_{k} \circ T_{k},
$$

и свободное правое действие группы $\operatorname{Aut}(\mathcal{F})$ на множестве $\mathbf{A F}$

$$
\mathbf{A F} \times \operatorname{Aut}(\mathcal{F}) \rightarrow \mathbf{A F}, \quad\left(\mathbf{r}_{k}, \boldsymbol{\rho}\right) \mapsto \mathbf{r}_{k} \cdot \boldsymbol{\rho}=\mathbf{r}_{k} \circ T_{k} \circ \boldsymbol{\rho} \circ T_{-k} .
$$

На группе (с поточечной групповой операцией)

$$
\begin{aligned}
\mathcal{M}(\boldsymbol{\Pi}) & =\left\{\mathbf{r} \in \operatorname{Map}(\mathbb{L} ; \mathbf{A F}) \mid \mathbf{\Pi} \circ \mathbf{r}=\operatorname{id}_{\mathbb{L}}\right\}= \\
& =\left\{\mathbf{r}: \mathbb{L} \rightarrow \mathbf{A F}, k \mapsto \mathbf{r}_{k} \in \operatorname{Aut}\left(\mathcal{F}_{k}\right)\right\}
\end{aligned}
$$

всех сечений расслоения $\mathbf{\Pi}: \mathbf{A F} \rightarrow \mathbb{L}$ группа $\mathbb{L}$ действует по правилу

$$
\begin{aligned}
T: \mathbb{L} \rightarrow \operatorname{Aut}(\mathcal{M}(\boldsymbol{\Pi})), \quad i \mapsto T_{i}: \mathcal{M}(\mathbf{\Pi}) & \rightarrow \mathcal{M}(\mathbf{\Pi}), \\
\mathbf{r} & \mapsto T_{i} \mathbf{r}=T_{-i} \circ \mathbf{r} \circ T_{i},
\end{aligned}
$$


так что здесь $k \mapsto\left(T_{i} \mathbf{r}\right)_{k}=T_{-i} \mathbf{r}_{k+i}=T_{-i} \circ \mathbf{r}_{k+i} \circ T_{i}$ для всех $i, k \in \mathbb{L}$. Подгруппа всех инвариантных сечений есть

$$
\begin{aligned}
\mathcal{M}_{T}(\boldsymbol{\Pi}) & =\left\{\mathbf{r} \in \mathcal{M}(\boldsymbol{\Pi}) \mid T_{i} \mathbf{r}=\mathbf{r} \text { для всех } i \in \mathbb{L}\right\}= \\
& =\left\{\mathbf{r} \in \mathcal{M}(\mathbf{\Pi}) \mid \mathbf{r}_{k+i}=T_{i} \circ \mathbf{r}_{k} \circ T_{-i} \text { для всех } i, k \in \mathbb{L}\right\} .
\end{aligned}
$$

ПрЕДЛОЖЕНИЕ 5. Имеет место изоморфизм групп

$$
\mathcal{M}_{T}(\boldsymbol{\Pi}) \simeq \operatorname{Aut}(\mathcal{F}), \quad \mathbf{r} \mapsto \boldsymbol{\rho}=\mathbf{r}_{0},
$$

обратное отображение $\boldsymbol{\rho} \mapsto \mathbf{r}$ действует по правилу $\mathbf{r}_{k}=T_{k} \circ \boldsymbol{\rho} \circ T_{-k}$ для всех $k \in \mathbb{L}$.

ДокАзАтЕЛьСтво. Достаточно воспользоваться определениями и предыдущими выкладками.

Вернемся к группе $\operatorname{SYM}\left(\boldsymbol{\pi}_{\mathbf{F}}\right)$. Пусть $R=\left(R_{\mathbf{F}}, R_{\mathbb{L}}\right) \in \operatorname{Aut}_{T}\left(\boldsymbol{\pi}_{\mathbf{F}}\right)$, так что $\mathbb{L} \ni k \mapsto$ $R_{\mathbb{L}}(k) \in \mathbb{L}, \mathbf{F} \ni(k, \mathbf{u}) \mapsto R_{\mathbf{F}}(k, \mathbf{u})=(l, \mathbf{v}) \in \mathbf{F}$. Из условия $R_{\mathbb{L}} \circ \boldsymbol{\pi}_{\mathbf{F}}=\boldsymbol{\pi}_{\mathbf{F}} \circ R_{\mathbb{L}}$ следует, что $l=R_{\mathbb{L}}(k)$, а из условия $T_{i} \circ R_{\mathbb{L}}=R_{\mathbb{L}} \circ T_{i}$ для всех $i \in \mathbb{L}$ следует, что $R_{\mathbb{L}}(k)=T_{m} k$ для некоторого $m \in \mathbb{L}$, не зависящего от $k \in \mathbb{L}$. Итак, $R_{\mathbf{F}}(k, \mathbf{u})=\left(T_{m} k, \mathbf{v}\right)=$ $T_{m}\left(k, \mathbf{r}_{k}(\mathbf{u})\right)$, где $\mathbf{r}_{k}(\mathbf{u})=T_{-m} \mathbf{v}(k, \mathbf{u}) \in \mathcal{F}_{k}$. Другими словами, $R=T_{m} \circ R^{0}$, где $T_{m} \in \mathcal{T}$, a $R^{0}=\left(R_{\mathbf{F}}^{0}, R_{\mathbb{L}}^{0}\right) \in \operatorname{Aut}_{T}^{0}\left(\boldsymbol{\pi}_{\mathbf{F}}\right), R_{\mathbf{F}}^{0}(k, \mathbf{u})=\left(k, \mathbf{r}_{k}(\mathbf{u})\right)$, где подгруппа

$$
\operatorname{Aut}_{T}^{0}\left(\boldsymbol{\pi}_{\mathbf{F}}\right)=\left\{R^{0}=\left(R_{\mathbf{F}}^{0}, R_{\mathbb{L}}^{0}\right) \in \operatorname{Aut}_{T}\left(\boldsymbol{\pi}_{\mathbf{F}}\right) \mid R_{\mathbb{L}}^{0}=\mathrm{id}_{\mathbb{L}}\right\}
$$

В частности, справедливо представление $\operatorname{Aut}_{T}\left(\boldsymbol{\pi}_{\mathbf{F}}\right)=\mathcal{T} \times \operatorname{Aut}_{T}^{0}(\mathbf{F})$, и, значит, $\operatorname{SYM}\left(\boldsymbol{\pi}_{\mathbf{F}}\right)=\operatorname{Aut}_{T}\left(\boldsymbol{\pi}_{\mathbf{F}}\right) / \mathcal{T}=\operatorname{Aut}_{T}^{0}\left(\boldsymbol{\pi}_{\mathbf{F}}\right)$.

ПРЕДЛОЖЕНИЕ 6. Имеет место изоморфизм групп

$$
\operatorname{Aut}_{T}^{0}\left(\boldsymbol{\pi}_{\mathbf{F}}\right) \simeq \mathcal{M}_{T}(\boldsymbol{\Pi})
$$

ДокАЗАтЕЛьство. Действительно, пусть $R^{0}=\left(R_{\mathbf{F}}^{0}, \mathrm{id}_{\mathbb{L}}\right) \in \operatorname{Aut}_{T}^{0}\left(\boldsymbol{\pi}_{\mathbf{F}}\right)$, где $R_{\mathbf{F}}^{0}(k, \mathbf{u})=\left(k, \mathbf{r}_{k}(\mathbf{u})\right), \mathbf{r}_{k}(\mathbf{u}) \in \mathcal{F}_{k}$, для всех $k \in \mathbb{L}, \mathbf{u} \in \mathcal{F}_{k}$. Легко проверяется, что для каждого $k \in \mathbb{L}$ правило $\mathbf{u} \mapsto \mathbf{r}_{k}(\mathbf{u})$ определяет автоморфизм $\mathbf{r}_{k} \in \operatorname{Aut}\left(\mathcal{F}_{k}\right)$, а затем правило $k \mapsto \mathbf{r}_{k}$ определяет сечение $\mathbf{r} \in \mathcal{M}(\boldsymbol{\Pi})$. Более того, в силу условия инвариантности $T_{i} \circ R_{\mathbf{F}}^{0}=R_{\mathbf{F}}^{0} \circ T_{i}$ для всех $i \in \mathbb{L}$ это сечение инвариантное, т. е. $\mathbf{r} \in \mathcal{M}_{T}(\boldsymbol{\Pi})$. Таким образом, определено отображение $\operatorname{Aut}_{T}^{0}\left(\boldsymbol{\pi}_{\mathbf{F}}\right) \rightarrow \mathcal{M}_{T}(\boldsymbol{\Pi}), R^{0} \mapsto \mathbf{r}$. Пусть теперь $\mathbf{r} \in \mathcal{M}_{T}(\boldsymbol{\Pi})$. Для каждого $(k, \mathbf{u}) \in \mathbf{F}$ положим $R_{\mathbf{F}}^{0}(k, \mathbf{u})=\left(k, \mathbf{r}_{k}(\mathbf{u})\right)$. Легко проверяется, что пара $R^{0}=\left(R_{\mathbf{F}}^{0}, \mathrm{id}_{\mathbb{L}}\right)$ задает автоморфизм расслоения $\boldsymbol{\pi}_{\mathbf{F}}: \mathbf{F} \rightarrow \mathbb{L}$ и что этот автоморфизм инвариантный, т. е. $R^{0} \in \mathrm{Aut}_{T}^{0}\left(\boldsymbol{\pi}_{\mathbf{F}}\right)$. Таким образом, определено отображение $\mathcal{M}_{T}(\boldsymbol{\Pi}) \rightarrow \operatorname{Aut}_{T}\left(\boldsymbol{\pi}_{\mathbf{F}}\right), \mathbf{r} \mapsto R^{0}$. По построению эти два отображения взаимно обратные, т. е. требуемый изоморфизм предъявлен.

ТЕОРема 2. Имеет место изоморфизм групп

$$
\operatorname{SYM}\left(\boldsymbol{\pi}_{\mathbf{F}}\right) \simeq \operatorname{Aut}(\mathcal{F})
$$

ДокАЗАТЕЛЬство. Достаточно воспользоваться установленным выше равенством $\operatorname{SYM}\left(\boldsymbol{\pi}_{\mathbf{F}}\right)=\operatorname{Aut}_{T}^{0}\left(\boldsymbol{\pi}_{\mathbf{F}}\right)$ и предложениями 6 и 5. 
2.5. Анализ на инвариантных подмножествах. Пусть $\mathbf{F}$ - инвариантное подмножество пространства $\mathbf{J}$.

На алгебре $\operatorname{Map}(\mathbf{F} ; \mathbb{R})$ определено действие $T$ группы $\mathbb{L}$ стандартным правилом

$$
\begin{aligned}
T: \mathbb{L} \rightarrow \operatorname{Aut}(\operatorname{Map}(\mathbf{F} ; \mathbb{R})), \quad i \mapsto T_{i}: \operatorname{Map}(\mathbf{F} ; \mathbb{R}) & \rightarrow \operatorname{Map}(\mathbf{F} ; \mathbb{R}), \\
f & \mapsto T_{i} f=f \circ T_{i},
\end{aligned}
$$

так что $\left(T_{i} f\right)(k, \mathbf{u})=f\left(T_{i} k, T_{i} \mathbf{u}\right)$ для всех $(k, \mathbf{u}) \in \mathbf{F}$.

Для каждого сечения $\boldsymbol{\phi} \in \mathcal{M}\left(\boldsymbol{\pi}_{\mathbf{F}}\right)$ определено отображение (подстановка)

$$
\left.\right|_{\phi}: \operatorname{Map}(\mathbf{F} ; \mathbb{R}) \rightarrow \mathcal{A}(\mathbb{L}),\left.\quad f \mapsto f\right|_{\phi},\left.\quad f\right|_{\phi}(k)=f(k, \phi(k)), \quad k \in \mathbb{L} .
$$

ПреДЛОЖЕНИЕ 7. Для любых $i \in \mathbb{L}, f \in \operatorname{Map}(\mathbf{F} ; \mathbb{R})$ u $\varphi \in \mathcal{M}_{\mathbf{F}}(\pi)$ выполняется правило полного сдвига

$$
T_{i}\left(\left.f\right|_{\mathbf{j} \varphi}\right)=\left.\left(T_{i} f\right)\right|_{\mathbf{j} \varphi} .
$$

ДокАЗАТЕЛЬство. Действительно, в этом случае для каждого $k \in \mathbb{L}$ имеем

$$
\left(T_{i}\left(\left.f\right|_{\mathbf{j} \varphi}\right)\right)(k)=\left(\left.f\right|_{\mathbf{j} \varphi}\right)\left(T_{i} k\right)=f\left(T_{i} k,(\mathbf{j} \varphi)\left(T_{i} k\right)\right)=f\left(T_{i} k, T_{i}((\mathbf{j} \varphi)(k))\right)=\left(\left.\left(T_{i} f\right)\right|_{\mathbf{j} \varphi}\right)(k),
$$

поскольку в силу предложения 3 сечение $\mathbf{j} \varphi$ инвариантное, и, значит, $T_{i} \circ(\mathbf{j} \varphi)=$ $(\mathbf{j} \varphi) \circ T_{i}$.

Пусть задана подалгебра $\mathcal{A}(\mathbf{F})$ алгебры $\operatorname{Map}(\mathbf{F} ; \mathbb{R})$ со следующими свойствами:

а) $\mathcal{A}(\mathbb{L}) \subset \mathcal{A}(\mathbf{F})$, в частности $\mathcal{A}(\mathbf{F})$ имеет структуру $\mathcal{A}(\mathbb{L})$-модуля;

б) алгебра $\mathcal{A}(\mathbf{F})$ инвариантна относительно сдвигов, $T_{i} \mathcal{A}(\mathbf{F})=\mathcal{A}(\mathbf{F})$ для всех $i \in \mathbb{L}$, т. е. действие $T: \mathbb{L} \rightarrow \operatorname{Aut}(\operatorname{Map}(\mathbf{F} ; \mathbb{R}))$ имеет сужение $T=\left.T\right|_{\mathcal{A}(\mathbf{F})}: \mathbb{L} \rightarrow \operatorname{Aut}(\mathcal{A}(\mathbf{F}))$.

В алгебре $\mathcal{A}(\mathbf{F})$ выделим подалгебру

$$
\mathcal{A}_{T}(\mathbf{F})=\left\{f \in \mathcal{A}(\mathbf{F}) \mid T_{i} f=f \text { для всех } i \in \mathbb{L}\right\}
$$

всех инвариантных функций (класса $\mathcal{A}(\mathbf{F}))$.

Определена алгебра Ли

$$
\mathfrak{D}(\mathcal{A}(\mathbf{F}))=\{X \in \mathfrak{g l}(\mathcal{A}(\mathbf{F})) \mid X(f \cdot g)=(X f) \cdot g+f \cdot(X g), f, g \in \mathcal{A}(\mathbf{F})\}
$$

всех дифференцирований алгебры $\mathcal{A}(\mathbf{F})$. На множестве $\mathfrak{D}(\mathcal{A}(\mathbf{F}))$ определена структура $\mathcal{A}(\mathbf{F})$-модуля,

$$
\mathcal{A}(\mathbf{F}) \times \mathfrak{D}(\mathcal{A}(\mathbf{F})) \rightarrow \mathfrak{D}(\mathcal{A}(\mathbf{F})), \quad(f, X) \mapsto f \cdot X
$$

согласованная со структурой алгебры Ли, где $(f \cdot X) g=f \cdot(X g)$ для всех $f, g \in \mathcal{A}(\mathbf{F})$, $X \in \mathfrak{D}(\mathcal{A}(\mathbf{F}))$.

ПреДЛОЖЕНИЕ 8. Сужение $\left.\mathfrak{D}(\mathcal{A}(\mathbf{F}))\right|_{\mathcal{A}(\mathbb{L})}=0$, m.e. $X \varphi=0$ для всех $X \in$ $\mathfrak{D}(\mathcal{A}(\mathbf{F})) u \varphi \in \mathcal{A}(\mathbb{L})$.

ДоказАтЕльство. Для каждого $l \in \mathbb{L}$ определена функция $\delta_{l} \in \mathcal{A}(\mathbb{L})$ правилом $\delta_{l}(k)=\delta_{l}^{k}$ (здесь $\delta_{l}^{k}-$ символ Кронекера), $k \in \mathbb{L}$. Пусть $X \in \mathfrak{D}(\mathcal{A}(\mathbf{F}))$. В силу правила Лейбница, $X \delta_{l}=X\left(\delta_{l} \cdot \delta_{l}\right)=2 \delta_{l} \cdot X \delta_{l}$, так что $X \delta_{l}=c_{l} \cdot \delta_{l}$, где функция $c_{l} \in \operatorname{Map}\left(\mathcal{F}_{l} ; \mathbb{R}\right)$, причем $c_{l} \cdot \delta_{l}=2 c_{l} \cdot \delta_{l} \cdot \delta_{l}=2 c_{l} \cdot \delta_{l}$, т. е. $c_{l}=0$, и, следовательно, $X \delta_{l}=0$ 
для всех $l \in \mathbb{L}$. Пусть $\varphi \in \mathcal{A}(\mathbb{L})$, тогда справедливо представление $\varphi=\sum_{l \in \mathbb{L}} \varphi(l) \cdot \delta_{l}$, так что

$$
X \varphi=X\left(\sum_{l \in \mathbb{L}} \varphi(l) \cdot \delta_{l}\right)=\sum_{l \in \mathbb{L}} \varphi(l) \cdot X \delta_{l}=0
$$

(последний переход использует непрерывность дифференцирования $X$, предполагаемую по умолчанию, см. вводные замечания).

СЛЕДСТВИЕ 2. Имеется естественное вложение алгебр Ли

$$
\mathfrak{D}(\mathcal{A}(\mathbf{F})) \subset \mathfrak{g l}_{\mathcal{A}(\mathbb{L})}(\mathcal{A}(\mathbf{F}))
$$

На алгебре Ли $\mathfrak{D}(\mathcal{A}(\mathbf{F}))$ определено действие группы $\mathbb{L}$ правилом

$$
\begin{aligned}
T: \mathbb{L} \rightarrow \operatorname{Aut}(\mathfrak{D}(\mathcal{A}(\mathbf{F}))), \quad i & \mapsto T_{i}: \mathfrak{D}(\mathcal{A}(\mathbf{F})) \\
X & \mapsto \mathfrak{D}(\mathcal{A}(\mathbf{F})), \\
X & \mapsto T_{i} X=T_{i} \circ X \circ T_{-i} .
\end{aligned}
$$

В алгебре Ли $\mathfrak{D}(\mathcal{A}(\mathbf{F}))$ выделим подалгебру

$$
\begin{aligned}
\mathfrak{D}_{T}(\mathcal{A}(\mathbf{F})) & =\left\{X \in \mathfrak{D}(\mathcal{A}(\mathbf{F})) \mid T_{i} X=X \text { для всех } i \in \mathbb{L}\right\}= \\
& =\left\{X \in \mathfrak{D}(\mathcal{A}(\mathbf{F})) \mid T_{i} \circ X=X \circ T_{i} \text { для всех } i \in \mathbb{L}\right\}
\end{aligned}
$$

всех инвариантных дифференцирований алгебры $\mathcal{A}(\mathbf{F})$.

ПРЕДЛОЖЕНИЕ 9. Определено сужение

$$
\left.\right|_{\mathcal{A}_{T}(\mathbf{F})}: \mathfrak{D}_{T}(\mathcal{A}(\mathbf{F})) \rightarrow \mathfrak{D}\left(\mathcal{A}_{T}(\mathbf{F})\right),\left.\quad X \mapsto X\right|_{\mathcal{A}_{T}(\mathbf{F})}
$$

где $\mathfrak{D}\left(\mathcal{A}_{T}(\mathbf{F})\right)$ - алгебра Ли всех дифберенцирований алгебры $\mathcal{A}_{T}(\mathbf{F})$.

ДокАЗАтельство. Действительно, здесь $T_{i}(X f)=X\left(T_{i} f\right)=X f$ для всех $i \in \mathbb{L}$, $X \in \mathfrak{D}_{T}(\mathcal{A}(\mathbf{F}))$ и $f \in \mathcal{A}_{T}(\mathbf{F})$, т. е. $\left.X\right|_{\mathcal{A}_{T}(\mathbf{F})}: \mathcal{A}_{T}(\mathbf{F}) \rightarrow \mathcal{A}_{T}(\mathbf{F})$, что и требовалось.

Разностный комплекс решетки $\mathbb{L}$ расширяется до аналогичного комплекса инвариантного подмножества $\mathbf{F}$ следующим образом.

Определен $\mathcal{A}(\mathbf{F})$-модуль

$$
\mathcal{D}(\mathcal{A}(\mathbf{F}))=\mathcal{A}(\mathbf{F}) \otimes_{\mathcal{A}(\mathbb{L})} \mathcal{D}(\mathbb{L}) \subset \operatorname{End}_{\mathbb{R}}(\mathcal{A}(\mathbf{F}))
$$

с базисом $\left\{\Delta_{1}, \ldots, \Delta_{D}\right\} \subset \operatorname{End}_{\mathbb{R}}(\mathcal{A}(\mathbf{F}))$. Дуальный модуль

$$
\mathcal{D}^{*}(\mathcal{A}(\mathbf{F}))=\operatorname{Hom}_{\mathcal{A}(\mathbf{F})}(\mathcal{D}(\mathbf{F}) ; \mathcal{A}(\mathbf{F}))=\mathcal{A}(\mathbf{F}) \otimes_{\mathcal{A}(\mathbb{L})} \mathcal{D}^{*}(\mathbb{L})
$$

обладает дуальным базисом $\left\{\Delta^{1}, \ldots, \Delta^{D}\right\}$. Группа $\mathbb{L}$ действует на линейных пространствах по-прежнему покомпонентно, причем для всех $i \in \mathbb{L}, \varrho \in \mathcal{D}^{*}(\mathcal{A}(\mathbf{F}))$, $\zeta \in \mathcal{D}(\mathcal{A}(\mathbf{F}))$ справедливо равенство $T_{i}\langle\varrho, \zeta\rangle=\left\langle T_{i} \varrho, T_{i} \zeta\right\rangle$.

Определена градуированная внешняя алгебра

$$
\Omega(\mathcal{A}(\mathbf{F}))=\bigoplus_{0 \leqslant q \leqslant D} \Omega^{q}(\mathcal{A}(\mathbf{F}))
$$


где $\mathcal{A}(\mathbf{F})$-модули

$$
\Omega^{q}(\mathcal{A}(\mathbf{F}))=\operatorname{Hom}_{\mathcal{A}(\mathbf{F})}\left(\bigwedge_{\mathcal{A}(\mathbf{F})}^{q} \mathcal{D}(\mathcal{A}(\mathbf{F})) ; \mathcal{A}(\mathbf{F})\right)=\mathcal{A}(\mathbf{F}) \otimes_{\mathcal{A}(\mathbb{L})} \Omega^{q}(\mathbb{L}) .
$$

Действие группы $\mathbb{L}$ на градуированной внешней алгебре $\Omega(\mathcal{A}(\mathbf{F}))$ по-прежнему покомпонентное. В частности, по-прежнему определены элементарные разностные операторы $\Delta_{\mu} \in \operatorname{End}_{\mathbb{R}}(\Omega(\mathcal{A}(\mathbf{F}))), \mu \in \mathbf{D}$.

По-прежнему разностный дифференциал $d \in \operatorname{End}_{\mathbb{R}}(\Omega(\mathcal{A}(\mathbf{F})))$ действует по правилу $\varrho \mapsto d \varrho=\sum_{\mu \in \mathbf{D}} \Delta^{\mu} \wedge \Delta_{\mu} \varrho$ для всех $\varrho \in \Omega(\mathcal{A}(\mathbf{F}))$, причем $d \circ d=0$ и $d^{q}=$ $\left.d\right|_{\Omega^{q}(\mathcal{A}(\mathbf{F}))}: \Omega^{q}(\mathcal{A}(\mathbf{F})) \rightarrow \Omega^{q+1}(\mathcal{A}(\mathbf{F}))$ для всех $0 \leqslant q \leqslant D$.

Итак, определен комплекс линейных пространств $\left\{\Omega^{q}(\mathcal{A}(\mathbf{F})) ; d^{q}\right\}-$ разностный комплекс инвариантного подмножества $\mathbf{F}$ (в классе $\mathcal{A}(\mathbf{F}))$.

Для каждого $\phi \in \mathcal{M}\left(\boldsymbol{\pi}_{\mathbf{F}}\right)$ подстановка $\left.\right|_{\phi}: \mathcal{A}(\mathbf{F}) \rightarrow \mathcal{A}(\mathbb{L})$ индуцирует покомпонентную подстановку $\left.\right|_{\phi}: \Omega(\mathcal{A}(\mathbf{F})) \rightarrow \Omega(\mathbb{L})$, в силу правила полного сдвига коммутирующую с разностным дифференциалом, т. е. определена следующая коммутативная диаграмма:

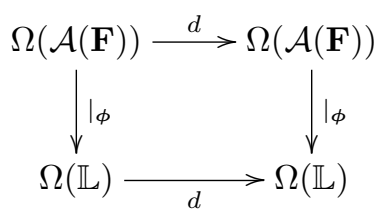

2.6. Факторизующие координаты. Пусть, как и в п $2.5, \mathbf{F}$ - инвариантное подмножество, $\mathcal{A}(\mathbf{F})$ - алгебра функций на $\mathbf{F}$ с указанными выше свойствами. Согласно предложению 2 имеет место изоморфизм расслоений

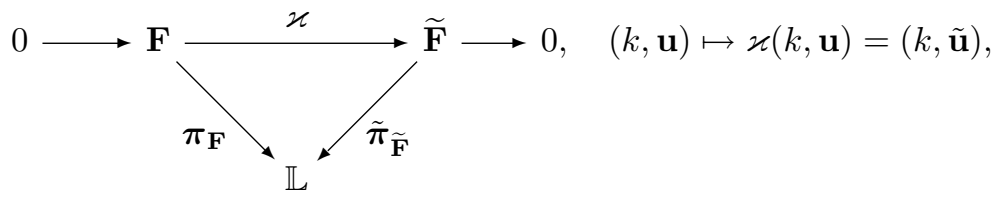

где $\widetilde{\mathbf{F}}=\mathbb{L} \times \mathcal{F}, \tilde{\mathbf{u}}=T_{-k} \mathbf{u}, \boldsymbol{\pi}_{\mathbf{F}}(k, \mathbf{u})=k=\tilde{\boldsymbol{\pi}}_{\widetilde{\mathbf{F}}}(k, \tilde{\mathbf{u}})$.

Будем рассматривать этот изоморфизм как глобальную карту, переменные $(k, \mathbf{u})-$ как точки пространства $\mathbf{F}$, а переменные $(k, \tilde{\mathbf{u}})-$ как их координаты на $\widetilde{\mathbf{F}}$. При такой интерпретации все конструкции п. 2.5 стандартным образом приобретают координатное представление.

Прежде всего отметим, что действие $T: \mathbb{L} \rightarrow \operatorname{Aut}(\mathbf{F})$ переходит в действие $\widetilde{T}=$ $\varkappa \circ T \circ \varkappa^{-1}: \mathbb{L} \rightarrow \operatorname{Aut}(\widetilde{\mathbf{F}})$, где, как легко проверить,

$$
\widetilde{T}_{i}(k, \tilde{\mathbf{u}})=\left(T_{i} k, \tilde{\mathbf{u}}\right)=(k+i, \tilde{\mathbf{u}}) \text { для всех } i, k \in \mathbb{L}, \quad \tilde{\mathbf{u}} \in \mathcal{F} .
$$

Таким образом, в координатном представлении сдвиги действуют только на целочисленные координаты. В этом и состоит его главное преимущество.

Изоморфизм $\varkappa: \mathbf{F} \simeq \widetilde{\mathbf{F}}$ индуцирует изоморфизм

$$
\varkappa^{*}: \operatorname{Map}(\widetilde{\mathbf{F}} ; \mathbb{R}) \simeq \operatorname{Map}(\mathbf{F} ; \mathbb{R}), \quad \tilde{f} \mapsto f=\varkappa^{*} \tilde{f}=\tilde{f} \circ \varkappa,
$$

так что $f(k, \mathbf{u})=\tilde{f}(k, \tilde{\mathbf{u}})=\tilde{f}\left(k, T_{-k} \mathbf{u}\right)$ для всех $(k, \mathbf{u}) \in \mathbf{F}$. 
На алгебре $\operatorname{Map}(\widetilde{\mathbf{F}} ; \mathbb{R})$ группа $\mathbb{L}$ действует по индуцированному правилу

$$
\begin{aligned}
\widetilde{T}: \mathbb{L} \rightarrow \operatorname{Aut}(\operatorname{Map}(\widetilde{\mathbf{F}} ; \mathbb{R})), \quad i \mapsto \widetilde{T}_{i}: \operatorname{Map}(\widetilde{\mathbf{F}} ; \mathbb{R}) & \rightarrow \operatorname{Map}(\widetilde{\mathbf{F}} ; \mathbb{R}), \\
\tilde{f} & \mapsto \widetilde{T}_{i} \tilde{f}=\tilde{f} \circ \widetilde{T}_{i},
\end{aligned}
$$

так что $\left(\widetilde{T}_{i} \tilde{f}\right)(k, \tilde{\mathbf{u}})=\tilde{f}\left(T_{i} k, \tilde{\mathbf{u}}\right)=\tilde{f}(k+i, \tilde{\mathbf{u}})$ для всех $(k, \tilde{\mathbf{u}}) \in \widetilde{\mathbf{F}}$.

Положим $\mathcal{A}(\widetilde{\mathbf{F}})=\left(\varkappa^{*}\right)^{-1} \mathcal{A}(\mathbf{F})$. Очевидно, $\mathcal{A}(\mathbb{L})=\left(\varkappa^{*}\right)^{-1} \mathcal{A}(\mathbb{L}) \subset \mathcal{A}(\widetilde{\mathbf{F}})$, и на алгебре $\mathcal{A}(\widetilde{\mathbf{F}})$ определено действие $\widetilde{T}$ группы $\mathbb{L}$. По построению $\varkappa^{*}: \mathcal{A}(\widetilde{\mathbf{F}}) \simeq \mathcal{A}(\mathbf{F})$.

Алгебра инвариантных функций $\mathcal{A}_{T}(\mathbf{F})$ имеет образ

$$
\mathcal{A}_{\widetilde{T}}(\widetilde{\mathbf{F}})=\left(\varkappa^{*}\right)^{-1} \mathcal{A}_{T}(\mathbf{F})=\left\{\tilde{f} \in \mathcal{A}(\widetilde{\mathbf{F}}) \mid \widetilde{T}_{i} \tilde{f}=\tilde{f}, i \in \mathbb{L}\right\}=\mathcal{A}(\mathcal{F}),
$$

т. е. состоит из всех функций $\tilde{f} \in \mathcal{A}(\widetilde{\mathbf{F}})$, не зависящих от целочисленных переменных $k \in \mathbb{L}$, и отождествляется с индуцированной алгеброй $\mathcal{A}(\mathcal{F})$ функций на типичном слое $\mathcal{F}$.

Изоморфизм $\varkappa^{*}: \mathcal{A}(\widetilde{\mathbf{F}}) \simeq \mathcal{A}(\mathbf{F})$ индуцирует изоморфизм алгебр Ли

$$
\varkappa_{*}: \mathfrak{D}(\mathcal{A}(\mathbf{F})) \simeq \mathfrak{D}(\mathcal{A}(\widetilde{\mathbf{F}})), \quad X \mapsto \widetilde{X}=\varkappa_{*} X=\left(\varkappa^{*}\right)^{-1} \circ X \circ \varkappa^{*} .
$$

На алгебре Ли $\mathfrak{D}(\mathcal{A}(\widetilde{\mathbf{F}}))$ определено действие группы $\mathbb{L}$ правилом

$$
\begin{aligned}
\widetilde{T}: \mathbb{L} \rightarrow \operatorname{Aut}(\mathfrak{D}(\mathcal{A}(\widetilde{\mathbf{F}}))), \quad i & \mapsto \widetilde{T}_{i}: \mathfrak{D}(\mathcal{A}(\widetilde{\mathbf{F}})) \\
\widetilde{X} & \mapsto \widetilde{T}_{i}(\mathcal{X}(\widetilde{\mathbf{F}})), \\
\widetilde{T} & \circ \widetilde{X} \circ \widetilde{T}_{-i} .
\end{aligned}
$$

Образ алгебры Ли $\mathfrak{D}_{T}(\mathcal{A}(\mathbf{F}))$ есть

$$
\mathfrak{D}_{\widetilde{T}}(\mathcal{A}(\widetilde{\mathbf{F}}))=\varkappa_{*} \mathfrak{D}_{T}(\mathcal{A}(\mathbf{F}))=\left\{\tilde{X} \in \mathfrak{D}(\mathcal{A}(\widetilde{\mathbf{F}})) \mid \widetilde{T}_{i} \tilde{X}=\tilde{X} \text { для всех } i \in \mathbb{L}\right\}
$$

- алгебра Ли всех инвариантных дифференцирований алгебры $\mathcal{A}(\widetilde{\mathbf{F}})$.

В частности, справедливо

ПРЕДЛОЖЕНИЕ 10. Имеет место изоморфизм

$$
\varkappa_{*}: \mathfrak{D}_{T}(\mathcal{A}(\mathbf{F})) \simeq \mathfrak{D}_{\widetilde{T}}(\mathcal{A}(\widetilde{\mathbf{F}})), \quad X \mapsto \widetilde{X}=\varkappa_{*} X=\left(\varkappa^{*}\right)^{-1} \circ X \circ \varkappa^{*} .
$$

Аналогичным образом, изоморфизм $\varkappa^{*}: \mathcal{A}(\widetilde{\mathbf{F}}) \simeq \mathcal{A}(\mathbf{F})$ индуцирует изоморфизмы $\mathcal{A}(\mathbb{L})$-модулей

$$
\begin{array}{ll}
\varkappa^{*}: \mathcal{D}(\mathcal{A}(\widetilde{\mathbf{F}})) \simeq \mathcal{D}(\mathcal{A}(\mathbf{F})), & \tilde{\zeta} \mapsto \zeta=\varkappa^{*} \tilde{\zeta}=\left(\varkappa^{*}\right) \circ \tilde{\zeta} \circ\left(\varkappa^{*}\right)^{-1}, \\
\varkappa^{*}: \mathcal{D}^{*}(\mathcal{A}(\widetilde{\mathbf{F}})) \simeq \mathcal{D}^{*}(\mathcal{A}(\mathbf{F})), & \tilde{\varrho} \mapsto \varrho=\varkappa^{*} \varrho=\varkappa^{*} \circ \tilde{\varrho} \circ\left(\varkappa^{*}\right)^{-1},
\end{array}
$$

причем $\varkappa^{*}\langle\tilde{\varrho}, \tilde{\zeta}\rangle=\left\langle\varkappa^{*} \tilde{\varrho}, \varkappa^{*} \tilde{\zeta}\right\rangle$ для всех $\tilde{\varrho} \in \mathcal{D}^{*}(\mathcal{A}(\widetilde{\mathbf{F}})), \tilde{\zeta} \in \mathcal{D}(\mathcal{A}(\widetilde{\mathbf{F}}))$. Группа $\mathbb{L}$ действует на линейных пространствах $\mathcal{D}(\mathcal{A}(\widetilde{\mathbf{F}}))$ и $\mathcal{D}^{*}(\mathcal{A}(\widetilde{\mathbf{F}}))$ покомпонентно, и $\widetilde{T}_{i}\langle\tilde{\varrho}, \tilde{\zeta}\rangle=$ $\left\langle\widetilde{T}_{i} \tilde{\varrho}, \widetilde{T}_{i} \tilde{\zeta}\right\rangle$ для всех $i \in \mathbb{L}, \tilde{\varrho} \in \mathcal{D}^{*}(\mathcal{A}(\widetilde{\mathbf{F}}))$ и $\tilde{\zeta} \in \mathcal{D}(\mathcal{A}(\widetilde{\mathbf{F}}))$.

В свою очередь, определен изоморфизм комплексов линейных пространств

$$
\varkappa^{*}:\left\{\Omega^{q}(\mathcal{A}(\widetilde{\mathbf{F}})) ; \tilde{d}^{q}\right\} \simeq\left\{\Omega^{q}(\mathcal{A}(\mathbf{F})) ; d^{q}\right\},
$$

дифференциал $\tilde{d} \in \operatorname{End}_{\mathbb{R}}(\Omega(\mathcal{A}(\widetilde{\mathbf{F}})))$ действует по тому же правилу, что и в правой части: $\tilde{\varrho} \mapsto \tilde{d} \tilde{\varrho}=\sum_{\mu \in \mathbf{D}} \tilde{\Delta}^{\mu} \wedge \tilde{\Delta}_{\mu} \tilde{\varrho}$ для всех $\tilde{\varrho} \in \Omega(\mathcal{A}(\widetilde{\mathbf{F}}))$, где $\tilde{\Delta}_{\mu}=\widetilde{T}_{e_{\mu}}-\widetilde{T}_{0}$, так что $\left\langle\tilde{\Delta}^{\mu}, \tilde{\Delta}_{\nu}\right\rangle=\delta_{\nu}^{\mu}$ 
2.7. Выбор класса функций и вычисления. Выше мы считали, что на инвариантном подмножестве $\mathbf{F} \subset \mathbf{J}=\mathbb{L} \times \mathbf{V}$ задана алгебра $\mathcal{A}(\mathbf{F})$, содержащая подалгебру $\mathcal{A}(\mathbb{L})$ и инвариантная относительно сдвигов. Алгебра $\mathcal{A}(\widetilde{\mathbf{F}})$ в этом случае возникает как прообраз алгебры $\mathcal{A}(\mathbf{F})$ при изоморфизме $\varkappa^{*}: \operatorname{Map}(\widetilde{\mathbf{F}} ; \mathbb{R}) \simeq \operatorname{Map}(\mathbf{F} ; \mathbb{R})$. Однако, как это принято в дифференциальной геометрии, более естественно задавать алгебру функций в координатном представлении, т. е. на карте $\widetilde{\mathbf{F}}=\mathbb{L} \times \mathcal{F}$. Именно так мы теперь и поступим. Выберем подходящую унитальную алгебру функций $\mathcal{A}(\mathcal{F})$ на типичном слое $\mathcal{F}$ и положим $\mathcal{A}(\widetilde{\mathbf{F}})=\mathcal{A}(\mathbb{L}) \otimes_{\mathbb{R}} \mathcal{A}(\mathcal{F})$. Эта алгебра инвариантна относительно сдвигов и содержит алгебру $\mathcal{A}(\mathbb{L})$ в качестве подалгебры. Ее образ $\mathcal{A}(\mathbf{F})=\varkappa^{*} \mathcal{A}(\widetilde{\mathbf{F}})$ также обладает всеми требуемыми свойствами. Такой выбор замечателен тем, что он позволяет в явном виде описать алгебру Ли $\mathfrak{D}_{\widetilde{T}}(\mathcal{A}(\widetilde{\mathbf{F}}))$ инвариантных дифференцирований алгебры $\mathcal{A}(\widetilde{\mathbf{F}})$ и вычислить когомологии комплекса $\left\{\Omega^{q}(\mathcal{A}(\widetilde{\mathbf{F}})) ; \tilde{d}^{q}\right\}$.

ТеОрема 3. Пусть алгебра $\mathcal{A}(\widetilde{\mathbf{F}})=\mathcal{A}(\mathbb{L}) \otimes_{\mathbb{R}} \mathcal{A}(\mathcal{F})$, где $\mathcal{A}(\mathcal{F})$ - унитальная алгебра функиий на типичном слое $\mathcal{F}$. Имеет место изоморфизм

$$
\left.\right|_{\mathcal{A}(\mathcal{F})}: \mathfrak{D}_{\widetilde{T}}(\mathcal{A}(\widetilde{\mathbf{F}})) \simeq \mathfrak{D}(\mathcal{A}(\mathcal{F})), \quad \widetilde{X} \mapsto \zeta=\left.\widetilde{X}\right|_{\mathcal{A}(\mathcal{F})}, \quad \zeta \mapsto \widetilde{X}=\operatorname{id}_{\mathcal{A}(\mathbb{L})} \otimes \zeta
$$

ДоказАтельство. Сужение $\widetilde{X} \mapsto \zeta=\left.\widetilde{X}\right|_{\mathcal{A}(\mathcal{F})}$ определено в силу предложения 9, причем это сужение инъективное в силу выбора $\mathcal{A}(\widetilde{\mathbf{F}})=\mathcal{A}(\mathbb{L}) \otimes_{\mathbb{R}} \mathcal{A}(\mathcal{F})$. Его сюръективность следует из равенства $\left.\left(\operatorname{id}_{\mathcal{A}(\mathbb{L})} \otimes \zeta\right)\right|_{\mathcal{A}(\mathcal{F})}=\zeta$ для всех $\zeta \in \mathfrak{D}(\mathcal{A}(\mathcal{F}))$.

СлЕДСТВИЕ 3. В условиях теоремы 3 имеет место изоморфизм

$$
I=\left.\right|_{\mathcal{A}(\mathcal{F})} \circ \varkappa_{*}: \mathfrak{D}_{T}(\mathcal{A}(\mathbf{F})) \simeq \mathfrak{D}(\mathcal{A}(\mathcal{F})) .
$$

ДокАЗАТЕЛЬство. Достаточно воспользоваться предложением 10.

Теорема 4. Пусть алгебра $\mathcal{A}(\widetilde{\mathbf{F}})=\mathcal{A}(\mathbb{L}) \otimes_{\mathbb{R}} \mathcal{A}(\mathcal{F})$, где $\mathcal{A}(\mathcal{F})$ - унитальная алгебра функций на типичном слое $\mathcal{F}$. Пополненный комплекс

$$
0 \longrightarrow \mathcal{A}(\mathcal{F}) \longrightarrow \Omega^{0}(\mathcal{A}(\widetilde{\mathbf{F}})) \stackrel{\tilde{d}^{0}}{\longrightarrow} \Omega^{1}(\mathcal{A}(\widetilde{\mathbf{F}})) \stackrel{\tilde{d}^{1}}{\longrightarrow} \cdots \stackrel{\tilde{d}^{D-1}}{\longrightarrow} \Omega^{D}(\mathcal{A}(\widetilde{\mathbf{F}})) \longrightarrow 0
$$

точный, т.е. все его пространства когомологий тривиальные.

ДокАЗАТЕльство. По определению $\Omega^{q}(\mathcal{A}(\widetilde{\mathbf{F}}))=\mathcal{A}(\widetilde{\mathbf{F}}) \otimes_{\mathcal{A}(\mathbb{L})} \Omega^{q}(\mathbb{L})$. В нашем случае алгебра $\mathcal{A}(\widetilde{\mathbf{F}})=\mathcal{A}(\mathbb{L}) \otimes_{\mathbb{R}} \mathcal{A}(\mathcal{F})$, что приводит к представлению $\Omega^{q}(\mathcal{A}(\widetilde{\mathbf{F}}))=$ $\mathcal{A}(\mathcal{F}) \otimes_{\mathbb{R}} \Omega^{q}(\mathbb{L})$. В свою очередь, дифференциал здесь имеет вид $\tilde{d}^{q}=\operatorname{id}_{\mathcal{A}(\mathcal{F})} \otimes_{\mathbb{R}} d^{q}$, где

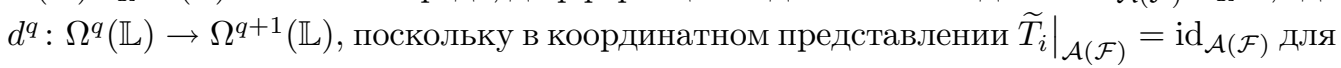
всех $i \in \mathbb{L}$. Таким образом, изучаемый комплекс в данной ситуации факторизуется, т. е. имеет место представление

$$
\left\{\Omega^{q}(\mathcal{A}(\widetilde{\mathbf{F}})) ; \tilde{d}^{q}\right\}=\mathcal{A}(\mathcal{F}) \otimes_{\mathbb{R}}\left\{\Omega^{q}(\mathbb{L}) ; d^{q}\right\} .
$$

Для завершения доказательства следует воспользоваться теоремой 1.

СлеДСтвиЕ 4. В условиях теоремы линейнъе пространства когомологий

$$
H^{q}(\mathcal{A}(\mathbf{F}))=\frac{\operatorname{Ker} d^{q}}{\operatorname{Im} d^{q-1}}= \begin{cases}\mathcal{A}(\mathcal{F}), & q=0 \\ 0, & q \neq 0\end{cases}
$$




\section{3. АЛГЕБРО-ГЕОМЕТРИЧЕСКИЙ АНАЛИЗ РАЗНОСТНЫХ СИСТЕМ}

3.1. Системы разностных уравнений. Будем рассматривать переменные $k \in \mathbb{L}$ как независимые, переменные $u \in V$ - как зависимые, а переменные $\mathbf{u} \in \mathbf{V}$ - как разностные.

Пусть $\mathcal{A}(\mathbf{J})$ - алгебра функций на пространстве $\mathbf{J}$, содержащая подалгебру $\mathcal{A}(\mathbb{L})$ и инвариантная относительно сдвигов. Система разностных уравнений на решетке $\mathbb{L}($ класса $\mathcal{A}(\mathbf{J}))$ есть система вида

$$
F^{\sigma}(k, \mathbf{u})=0, \quad \sigma \in \mathrm{S}, \quad(k, \mathbf{u}) \in \mathbf{J}
$$

(коротко $F=0)$, где семейство функций $F=\left(F^{\sigma}\right) \in \mathcal{A}(\mathbf{J})^{\mathrm{S}}, \mathrm{S}$ - некоторое множество индексов. По определению сечение $\varphi \in \mathcal{M}(\pi)$ есть решение системы $F=0$, если подстановка $\left.F\right|_{\mathbf{j} \varphi}=0$, подробнее

$$
F^{\sigma}(k, \mathbf{j} \varphi(k))=0 \quad \text { для всех } \sigma \in \mathrm{S}, \quad k \in \mathbb{L} .
$$

Множество всех решений системы $F=0$ есть

$$
\operatorname{Sol}\{F=0\}=\left\{\varphi \in \mathcal{M}(\pi)|F|_{\mathbf{j} \varphi}=0\right\} .
$$

По построению система $F=0$ задает подмножество

$$
\left\{(k, \mathbf{u}) \in \mathbf{J} \mid F^{\sigma}(k, \mathbf{u})=0 \text { для всех } \sigma \in \mathrm{S}\right\} \subset \mathbf{J},
$$

которое не является инвариантным и не пригодно для дальнейшего анализа, однако оно порождает инвариантное подмножество

$$
\mathbf{F}=\left\{(k, \mathbf{u}) \in \mathbf{J} \mid\left(T_{i} F^{\sigma}\right)(k, \mathbf{u})=0 \text { для всех } i \in \mathbb{L}, \sigma \in \mathbf{S}\right\} \subset \mathbf{J} .
$$

ПреДЛОЖЕНИЕ 11. Сечение $\varphi \in \mathcal{M}(\pi)$ есть решение разностной системы $F=0$ тогда и только тогда, когда оно допустимое для подрасслоения $\boldsymbol{\pi}_{\mathbf{F}}: \mathbf{F} \rightarrow \mathbb{L}$, m.е.

$$
\operatorname{Sol}\{F=0\}=\{\varphi \in \mathcal{M}(\pi) \mid \operatorname{graph} \mathbf{j} \varphi \subset \mathbf{F}\}=\mathcal{M}_{\mathbf{F}}(\pi)
$$

В частности, системь $\left\{F^{\sigma}=0 \mid \sigma \in \mathrm{S}\right\}$ u $\left\{T_{i} F^{\sigma}=0 \mid i \in \mathbb{L}, \sigma \in \mathrm{S}\right\}$ равносильнъ.

ДокАзАТЕЛЬСтво. Достаточно воспользоваться правилом полного сдвига, согласно которому $\left.\left(T_{i} F^{\sigma}\right)\right|_{\mathbf{j} \varphi}=T_{i}\left(\left.F^{\sigma}\right|_{\mathbf{j} \varphi}\right)$ для всех $i \in \mathbb{L}, \sigma \in \mathrm{S}$.

Инвариантное подмножество $\mathbf{F}$ является адекватным геометрическим образом разностной системы $F=0$, пригодным для алгебро-геометрического анализа свойств этой системы.

ТЕОРема 5. Имеет место канонический изоморфизм

$$
\operatorname{Sol}\{F=0\} \cong \mathcal{F}, \quad \varphi \mapsto \mathbf{u}, \quad u_{l}=\varphi(l) \quad \text { для всех } l \in \mathbb{L},
$$

позволяющий отождествлять множество $\operatorname{Sol}\{F=0\}$ и типичный слой

$$
\mathcal{F}=\mathcal{F}_{0}=\left\{\mathbf{u} \in \mathbf{V} \mid F^{\sigma}\left(i, T_{i} \mathbf{u}\right)=0 \text { для всех } \sigma \in \mathrm{S}, i \in \mathbb{L}\right\} .
$$

ДокАЗАТЕЛЬСтво. Достаточно воспользоваться предложениями 11 и 3. 
3.2. Симметрии и законы сохранения. Автоморфизмы множества $\operatorname{Sol}\{F=$ $0\}$ всех решений данной системы $F=0$ называются глобалъными симметриями этой системы. Множество всех таких симметрий образует группу $\operatorname{SYM}\{F=0\}=$ $\operatorname{Aut}(\operatorname{Sol}\{F=0\})$.

Теорема 6. Имеет место представление

$$
\operatorname{SYM}\{F=0\}=\operatorname{Aut}(\mathcal{F}) \simeq \operatorname{SYM}\left(\boldsymbol{\pi}_{\mathbf{F}}\right)
$$

ДокАЗАТЕЛьство. Достаточно воспользоваться теоремами 5 и 2.

Дальнейший анализ требует спецификации класса функций на множестве $\mathbf{F}$. Для этого, как было предложено в п. 2.7, выберем унитальную алгебру функций $\mathcal{A}(\mathcal{F})$, согласованную с исходной алгеброй $\mathcal{A}(\mathbf{J})$, и положим $\mathcal{A}(\mathbf{F})=\varkappa^{*} \mathcal{A}(\widetilde{\mathbf{F}})$, где $\mathcal{A}(\widetilde{\mathbf{F}})=$ $\mathcal{A}(\mathbb{L}) \otimes_{\mathbb{R}} \mathcal{A}(\mathcal{F})$

Теорема 6 и аналогия с алгебро-геометрическим анализом уравнений в частных производных позволяют считать дифференцирования алгебры $\mathcal{A}(\mathcal{F})$ инфинитезимальными симметриями системы $F=0$. Множество всех таких симметрий образует алгебру Ли $\operatorname{sym} F=0$.

Теорема 7. Имеет место представление

$$
\operatorname{sym}\{F=0\}=\mathfrak{D}(\mathcal{A}(\mathcal{F})) \simeq \mathfrak{D}_{T}(\mathcal{A}(\mathbf{F}))
$$

ДокАЗАтЕльство. Достаточно воспользоваться следствием 3.

Снова по аналогии с уравнениями в частных производных считаем когомологии разностного комплекса $\left\{\Omega^{q}(\mathcal{A}(\mathbf{F})) ; d^{q}\right\}$ законами сохранения разностной системы $F=0$. Обозначим через $\mathrm{CL}^{q}\{F=0\}=H^{q}(\mathcal{A}(\mathbf{F}))$ линейное пространство всех законов сохранения $q$-го порядка. Согласно следствию 4 справедлива

Теорема 8. Имеет место представление

$$
\mathrm{CL}^{q}\{F=0\}= \begin{cases}\mathcal{A}(\mathcal{F}), & q=0 \\ 0, & q \neq 0\end{cases}
$$

Таким образом, в данной постановке нетривиальные законы сохранения имеются лишь при $q=0$. В этом случае они называются интегралами системы $F=0$ и имеют описание

$$
\begin{aligned}
\mathrm{CL}^{0}\{F=0\} & =\left\{f(k, \mathbf{u}) \in \mathcal{A}(\mathbf{F})|f|_{\mathbf{j} \varphi}(k)=\mathrm{const} \quad \text { для всех } \varphi \in \operatorname{Sol}\{F=0\}\right\}= \\
& =\left\{f(k, \mathbf{u})=g\left(T_{-k} \mathbf{u}\right) \in \mathcal{A}_{T}(\mathbf{F}) \mid g(\mathbf{u}) \in \mathcal{A}(\mathcal{F})\right\} .
\end{aligned}
$$

Элементарные интеграль системы $F=0$ суть функции $f_{l} \in \mathcal{A}_{T}(\mathbf{F}), l \in \mathbb{L}$, где $f_{l}(k, \mathbf{u})=g_{l}\left(T_{-k} \mathbf{u}\right),(k, \mathbf{u}) \in \mathbf{F}, g_{l}(\tilde{\mathbf{u}})=\tilde{u}_{l}, \tilde{\mathbf{u}}=\left(\tilde{u}_{l}\right) \in \mathcal{F}\left(\right.$ коротко $\left.f_{l}(k, \mathbf{u})=u_{l-k}\right)$, так что

$$
\left.f_{l}\right|_{\mathbf{j} \varphi}(k)=f_{l}(k, \mathbf{j} \varphi(k))=\varphi(l) \quad \text { для всех } \varphi \in \operatorname{Sol}\{F=0\}, \quad k \in \mathbb{L} .
$$

В стандартной ситуации элементарные интегралы порождают алгебру $\mathrm{CL}^{0}\{F=0\}$. 


\section{4. ОБСУЖДЕНИЕ}

Очевидно, успех предложенной схемы алгебро-геометрического анализа данной разностной системы $F=0$ зависит от возможности конструктивного описания типичного слоя $\mathcal{F}$ и удачного выбора алгебры $\mathcal{A}(\mathcal{F})$. Идеальный вариант - задание на слое $\mathcal{F}$ структуры гладкого многообразия (как правило, бесконечномерного) и выбор в качестве $\mathcal{A}(\mathcal{F})$ алгебры всех гладких функций на $\mathcal{F}$ локалъно-конечного порядка (т. е. зависящих от конечного числа локальных координат). Можно привести простые модельные примеры, когда это практически осуществимо, но для большинства возникающих в приложениях разностных систем реализация такой идеальной ситуации достаточно сложна или невозможна. Здесь следует также отметить, что согласно теореме 5 конструктивное описание типичного слоя $\mathcal{F}$ технически совпадает с описанием множества всех решений системы $F=0$. В общем случае предлагается следующая конструкция.

Для каждого $p \in \mathbb{Z}_{+}$положим $\mathbf{V}_{p}=\left\{\mathbf{u}=\left(u_{i}\right)\left|u_{i} \in V,\right| i \mid \leqslant p\right\}$ и обозначим через $\mathcal{A}\left(\mathbf{V}_{p}\right)=\mathcal{C}^{\infty}\left(\mathbf{V}_{p}\right)$ алгебру всех гладких функций на евклидовом пространстве $\mathbf{V}_{p}$. Индуктивный предел $\mathcal{A}(\mathbf{V})=\lim _{p \rightarrow \infty} \mathcal{A}\left(\mathbf{V}_{p}\right)$ есть алгебра всех гладких функций конечного порлдка на пространстве $\mathbf{V}$, где порядок $p=p(f)$ функции $f=f(\mathbf{u}) \in$ $\mathcal{A}(\mathbf{J})$ определяется условием $f \in \mathcal{A}\left(\mathbf{V}_{p}\right) \backslash \mathcal{A}\left(\mathbf{V}_{p-1}\right)$. Группа $\mathbb{L}$ действует на алгебре $\mathcal{A}(\mathbf{V})$ стандартным образом:

$$
T: \mathbb{L} \rightarrow \operatorname{Aut}(\mathcal{A}(\mathbf{V})), \quad i \mapsto T_{i}: \mathcal{A}(\mathbf{V}) \rightarrow \mathcal{A}(\mathbf{V}), \quad f \mapsto T_{i} f=f \circ T_{i} .
$$

Тензорное произведение $\mathcal{A}(\mathbf{J})=\mathcal{A}(\mathbb{L}) \otimes_{\mathbb{R}} \mathcal{A}(\mathbf{V})$ есть алгебра всех гладких функций локально-конечного порядка на пространстве $\mathbf{J}=\mathbb{L} \times \mathbf{V}$, где порядок функции $f=$ $f(k, \mathbf{u}) \in \mathcal{A}(\mathbf{J})$ в точке $k \in \mathbb{L}$ определяется как порядок функции $g=g(\mathbf{u})=f(k, \mathbf{u}) \in$ $\mathcal{A}(\mathbf{V})$. Эта алгебра $\mathcal{A}(\mathbf{J})$ есть наиболее общая и естественная алгебра функций на $\mathbf{J}$, пригодная для алгебро-геометрического анализа систем разностных уравнений на решетке $\mathbb{L}$, и мы считаем, что изучаемая система имеет вид (см. начало раздела 3 )

$$
F^{\sigma}(k, \mathbf{u})=0, \quad \sigma \in \mathrm{S}, \quad(k, \mathbf{u}) \in \mathbf{J}, \quad F^{\sigma} \in \mathcal{A}(\mathbf{J}) .
$$

Типичный слой $\mathcal{F}=\left\{\mathbf{u} \in \mathbf{V} \mid F^{\sigma}\left(i, T_{i} \mathbf{u}\right)=0, \sigma \in \mathrm{S}, i \in \mathbb{L}\right\}$ (см. теорему 5) порождает идеал

$$
\mathcal{I}\{F=0\}=\left\{f \in \mathcal{A}(\mathbf{V})|f|_{\mathcal{F}}=0\right\}
$$

алгебры $\mathcal{A}(\mathbf{V})$, и мы принимаем в качестве алгебры функций на множестве $\mathcal{F}$ фактор-алгебру $\mathcal{A}(\mathcal{F})=\mathcal{A}(\mathbf{V}) / \mathcal{I}\{F=0\}$. Отметим, что подобные фактор-алгебры довольно часто применяются в практическом алгебро-геометрическом анализе уравнений в частных производных (вычисление симметрий, законов сохранения, преобразований Беклунда и т. д.), только этот факт явно не упоминается. Более того, если на множестве $\mathcal{F}$ имеется структура гладкого многообразия, согласованная с алгеброй $\mathcal{A}(\mathbf{J})$, то предлагаемая фактор-алгебра по существу совпадает с алгеброй всех гладких функций локально конечного порядка на многообразии $\mathcal{F}$. Дальнейшие построения следуют плану, изложенному в п. 3.2 , и принципиальных трудностей не вызывают.

Благодарности. Работа выполнена при частичной финансовой поддержке РФФИ (грант № 10-01-00178) и Программы поддержки ведущих научных школ (грант НШ-7675.2010.1). 


\section{Список литературы}

[1] Л. В. Овсянников, Групповой анализ дифференииалъных уравнений, Наука, М., 1978.

[2] В.Е. Захаров, С.В. Манаков, С. П. Новиков, Л.П. Питаевский, Теория солитонов. Метод обратной задачи, Наука, М., 1980.

[3] Н. Х. Ибрагимов, Группы преобразований в математической физике, Наука, М., 1983.

[4] А. М. Виноградов, И.С. Красильщик, В. В. Лычагин, Введение в геометрию нелинейных дифференииалъных уравнений, Наука, М., 1986.

[5] П. Олвер, Приложения групп Ли к дифференциалъным уравнениям, Мир, М., 1989.

[6] V.V. Zharinov, Lecture Notes on Geometrical Aspects of Partial Differential Equations, Ser. Sov. East Eur. Math., 9, World Sci., Singapore, 1992.

[7] А. М. Виноградов, И. С. Красильщик (ред.), Симметрии и законы сохранения уравнений математической физики, Факториал, М., 1997.

[8] B. Kupershmidt, Discrete Lax equations and differential-difference calculus, Astérisque, 123, SMF, Paris, 1985.

[9] Ü. Göktaş, W. Hereman, Physica D, 123:1-4 (1998), 425-436, arXiv: solv-int/9801023.

[10] В. Э. Адлер, А. Б. Шабат, Р. И. Ямилов, ТМФ, 125:3 (2000), 355-424.

[11] E. L. Mansfield, P. E. Hydon, "On a variational complex for difference equations", The Geometrical Study of Differential equations, Contemp. Math., 285, eds. J. A. Leslie, T. P. Robart, AMS, Providence, RI, 2002, 121-129.

[12] M.S. Hickman, W. A. Hereman, Proc. Roy. Soc. A, 459:2039 (2003), 2705-2729.

[13] P. E. Hydon, E. L. Mansfield, Found. Comput. Math., 4:2 (2004), 187-217.

[14] W. Hereman, M. Colagrosso, R. Sayers, A. Ringler, B. Deconinck, M. Nivala, M. Hickman, "Continuous and discrete homotopy operators and the computation of conservation laws", Differential Equations With Symbolic Computation, Trends Math., eds. D. Wang, Z. Zheng, Basel, Birkhäuser, 2005, 255-290.

[15] В. В. Жаринов, ТМФ, 157:3 (2008), 391-405.

[16] В. В. Жаринов, ТМФ, 165:2 (2010), 195-216.

[17] В. В. Жаринов, ТМФ, 144:3 (2005), 435-452.

Поступила в редакцию 3.02.2011 